\title{
Analysis on the Factors Causing the Real-Time Image Blurry and Development of Methods for the Image Restoration
}

\author{
Jianhua Zhang, Ronghua Ji, Kaiqun Hu, Xue Yuan, Hui Li, and Lijun Qi ${ }^{*}$ \\ College of Engineering, China Agricultural University, Beijing, 100083, P.R. China
}

\begin{abstract}
The blurred images will make image processing difficult and be hard to get high image solution for recognition, which will lower the precision of a variable rate spray system in pesticide application. In this paper, Radon transform in frequency domain is used in this work to test the blur angle and blur length in different tractors speeds and camera heights. Four image restoration methods in terms of the shape, color, texture and computing time were compared. The results showed that the speed of tractor and the height of camera played an important role on the blur extent of real-time image. A mathematical model corresponding blur angle and blur length with the speed of tractor and the height of camera is established, which would provide a theoretical basis for reducing blur and improving the quality of real-time image.
\end{abstract}

Keywords: Machine vision, Real-time, Blurred image, Radon transform, Image restoration.

\section{Introduction}

The images captured by the variable rate spraying system based on machine vision are often blurry in some extent due to the relative move between camera and objects as well as the vibration of the system in the field [2, 3, 5-10]. Motion blur will make image processing difficult and be hard to get high image solution for recognition, which will lower the precision for the system to adjust the spray volume in terms of the practices of the crops $[4,11,12]$. As the variable rate spraying system in the actual operation, how to reduce the blur of real-time image captured and restore the blurred image is crucial.

In the imaging process, image always appears blur due to the relative move between camera and objects, Imaging equipment with external interference and inherent defects, The vibration and light scattering of the camera, and noise interference [6]. The blurring needs to determine the point spread function, it can be expressed as:

$$
h(x, y)=\left\{\begin{array}{cc}
1 / L & \sqrt{x^{2}+y^{2} \leq L}, x / y=-\tan (\theta) \\
0 & \text { others }
\end{array}\right.
$$

\footnotetext{
${ }^{*}$ Corresponding author.
} 
Where $\mathrm{L}$ is blur length, $\theta$ is blur angle, they are two important parameters of motion blur [7]. The main methods of detecting blur angle and blur length are divided into differential method and frequency spectrum. Differentiation is the calculation of the direction of the process of differential reference only to two adjacent pixels of the gray value, not well reflect the changes in the gray image areas, and the result error is relatively large. Spectrum method is based on computation of the power spectrum of the image in the frequency domain, and the error is relatively low [8].

The image restoration methods have been matured, which are then based on deconvolution methods such as iterative Lucy-Richardson and Blind deconvolution or non-iterative Wiener algorithms and Regularized filter [9]. However, these real-time image restoration method to evaluate the need for restoration effect. Common image evaluation methods are: Mean Absolute Error(MAE), Mean Square Error (MSE), Normalized Mean Square Error (NMSE), Signal to Noise Ratio (SNR) and Peak Signal to Noise Ratio(PSNR) [10]. However, these methods are based on reference image quality assessment, and not meet the interest in evaluation of machine vision.

In this paper, mathematical models established for blurred image analysis through testing blur degree at the different speed of tractor and the height of camera, which provide a theoretical basis for reducing motion blur in images. Comparison of the four kinds of classical methods of image restoration such as Wiener filter, Regularized filter, Lucy Richardson (L-R) and Blind algorithm already in use and some which have been recently proposed in the literature for finding a restoration method suitable for real-time image.

\section{Experimental Materials and Methods}

\subsection{Experimental Equipment}

Real-time image acquisition system was based on a small four-wheel system DFH tractor as the carrier, and pre-high-resolution color camera for the OK_AC1300 RGB camera, 230 frames / second. Image acquisition card was OK_RGB10B RGB color component capture card. Lenovo notebook computer with Pentium4 / 2.6G processor, $512 \mathrm{M}$ memory was used. Speed sensor was DJRVS11 made by Spraying System Company, USA.

\subsection{Image Acquisition}

We had a trial in the experimental base for China Agricultural University in early June 2010, Cotton as the experimental object, which was seedling growth period and its height between $10 \sim 20 \mathrm{~cm}$. This was orthogonal experimental design, the heights of Camera were $50 \mathrm{~cm}, 80 \mathrm{~cm}, 110 \mathrm{~cm}, 140 \mathrm{~cm}$ and $170 \mathrm{~cm}$, tractor speeds were $2 \mathrm{~km} / \mathrm{h}$, $4 \mathrm{~km} / \mathrm{h}, 6 \mathrm{~km} / \mathrm{h}, 8 \mathrm{~km} / \mathrm{h}, 10 \mathrm{~km} / \mathrm{h}$ and $12 \mathrm{~km} / \mathrm{h}$ of 6 speed. We used real-time image acquisition system for image acquisition on cotton leaves, each collection was repeated 3 times, and stored the image acquainted through image acquisition card in the computer. Image acquisition card comes with the package can choose from 7 different image resolution for storage. 


\subsection{Motion Blurring Parameters Estimation}

When the gradient of such a blurred image is transformed into the frequency domain by the Fast Fourier Transform (FFT) a regular structure is contained within the power spectrum in the frequency domain. This regular structure has a relationship to the length and the direction of the blur. The distance between neighboring stripes is related to the length of the motion blur. The relationship between blur length $L$ and the distance between neighboring stripes $d$ can be simply expressed as: $L=a /(d+b)$. The main ideas of the finding blur parameters process are illustrated in Fig. 1.

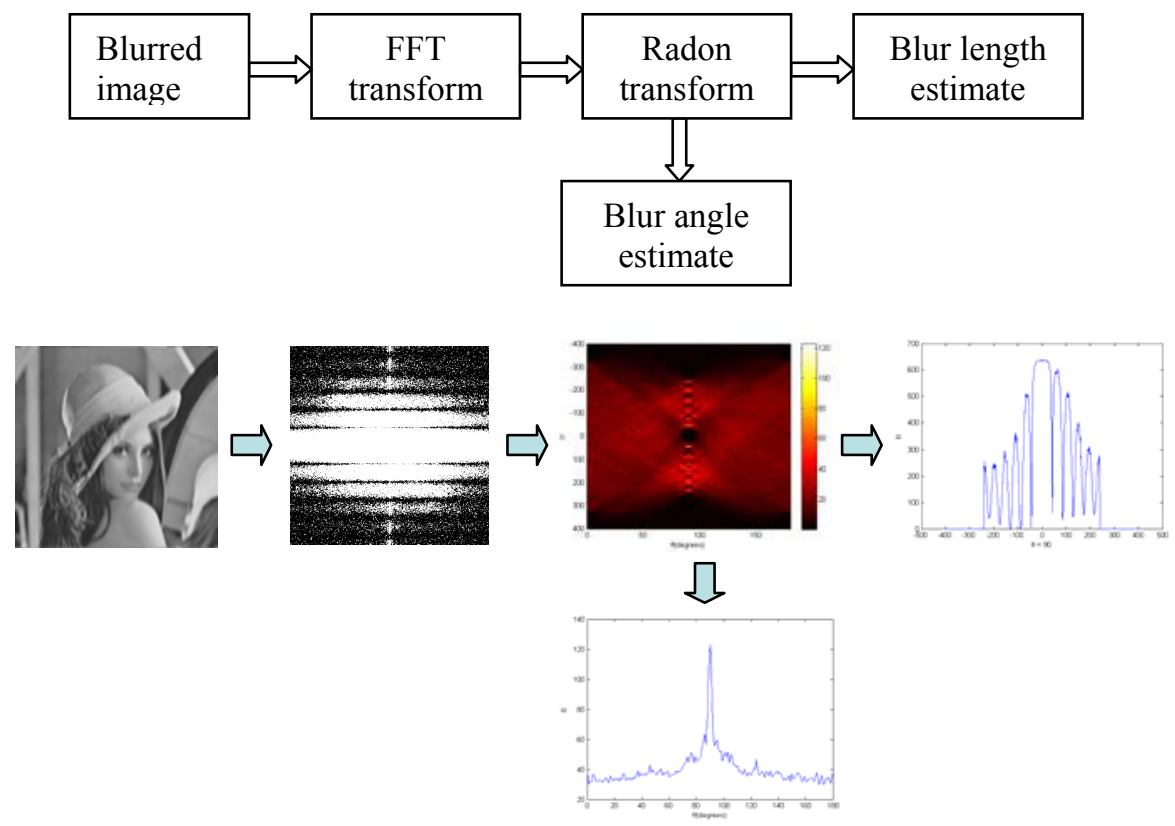

Fig. 1. The process of finding blurring parameters

\subsection{Image Restoration Methods}

(1) Wiener filter

The Wiener filter seeks to minimize the following error function:

$$
e^{2}=E\left\{(f-\hat{f})^{2}\right\}
$$

Where $E$ denotes the expected value operator, $f$ is the undegraded image and $\hat{f}$ its estimate. The solution to the thereof arising optimization task can be written as follows in the frequency domain:

$$
\hat{F}=\left[\frac{1}{H(u, v)} \frac{|H(u, v)|^{2}}{|H(u, v)|^{2}+S_{\eta}(u, v) / S_{t}(u, v)}\right] B
$$


Where $\mathrm{H}(\mathrm{u}, \mathrm{v})$ is the PSF in the frequency domain, $S_{\eta}(u, v)$ the power spectrum of the noise and $S_{t}(u, v)$ the power spectrum of the undegraded image $F$.

(2) Regularized filter (reg)

This algorithm [15] is based on finding a direct filter solution using a criterion $C$, which ensures optimal smoothness of the image restored. Therefore the filter construction task is to find the minimum of

$$
\begin{gathered}
C=\sum_{u=1}^{M} \sum_{v=1}^{N}\left[\nabla^{2} f(u, v)\right]^{2} \\
\|b-h * \hat{f}\|^{2}=\|n\|^{2}
\end{gathered}
$$

In the frequency domain the solution to this problem can be written as follows:

$$
\hat{F}(u, v)=\left[\frac{H^{*}(u, v)}{|H(u, v)|^{2}+\gamma|P(u, v)|^{2}}\right] B(u, v)
$$

Where $\gamma$ is the parameter which has to be adjusted to fulfill the constraint $C$ and $P(u, v)$ is the Laplacian operator in the frequency domain.

(3) Lucy-Richardson deconvolution (L-R)

This algorithm was invented independently by Lucy [14] and Richardson [15]. Its usage is further outline din [16]. The L-R algorithm is an iterative restoration algorithm that maximizes a Poisson statistics image model likelihood function. As summed up in [17] the RL algorithm consists of one initial and three iterative steps:

Step 1: A first approximation of the restored image $\hat{f_{0}}$ must be made, typically the constant average of all pixel values in the blurred image $b$.

Step 2: The current approximation is convolved with the PSF:

$$
\varphi_{n}=h * \hat{f}_{n}
$$

Step 3: A correction factor is computed based on the ratio of the blurred image and the result of the last step:

$$
\phi_{n}=\overleftarrow{h} * \frac{b}{\varphi_{n}}
$$

Where $\overleftarrow{h}$ denotes the PSF in reverse order and $b / \varphi_{n}$ a "pixel-by-pixel" division.

Step 4: A new approximate is composed out of the current one and the correction factor:

$$
\hat{f}_{n+1}=\hat{f}_{n} \cdot \phi_{n}
$$


(4) Blind deconvolution (Blind)

Blind deconvolution algorithm restores the original blurred image with estimating PSF. The solution improved through iteration, the likelihood of that increases in the number of iterations, eventually converge with the greatest likelihood of Solutions Division. The advantage of the algorithm is able to achieve the blurred image of the recovery operations, in the case of no prior knowledge [20].

\section{Experimental Results and Analysis}

\subsection{Analysis Blurred Factors of Image}

To minimizing the blur extent, need for analysis of blur factors. Tractor speed and the camera above the ground as the factors, we made the analysis of blur angle and blur length with 90 images, which were detected by Radon transform in frequency domain. We established the relationship between blur angle and tractor speed with camera height shown in Fig. 2, and the relationship between blur length and tractor speed with camera height shown in Fig. 3.

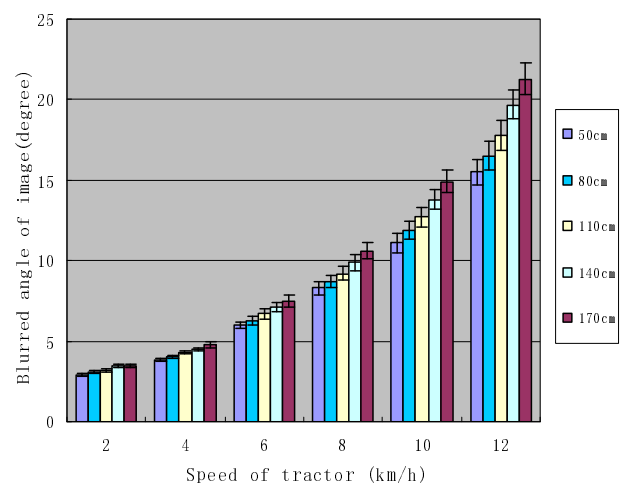

Fig. 2. The relationship of between tractor speed and blur angle

As can be seen from Fig. 2, engine vibration amplitude became larger and more increase degree of body bump with the increase of tractor speed. Therefore, Angle of deviation from the center line of tractors and the blur angle of image acquisition increased. Average blur angle increased from 3 degrees to 16 degrees as tractor speed increased from 2 to 12 . As the camera away from the higher ground, its body was shaking with the more severe. Therefore, with the increase of the height of camera, the image blur angle became more. Moreover, camera height greater impact on blur angle of image with tractor traveling increase, the average blur angle expand 0.6 degrees with camera height increase from $50 \mathrm{~cm}$ to $170 \mathrm{~cm}$ at the speed of 2 , while the average blur angle expand 5.2 degrees with camera height increase from $50 \mathrm{~cm}$ to $170 \mathrm{~cm}$ at the speed of $12 \mathrm{~km} / \mathrm{h}$. 


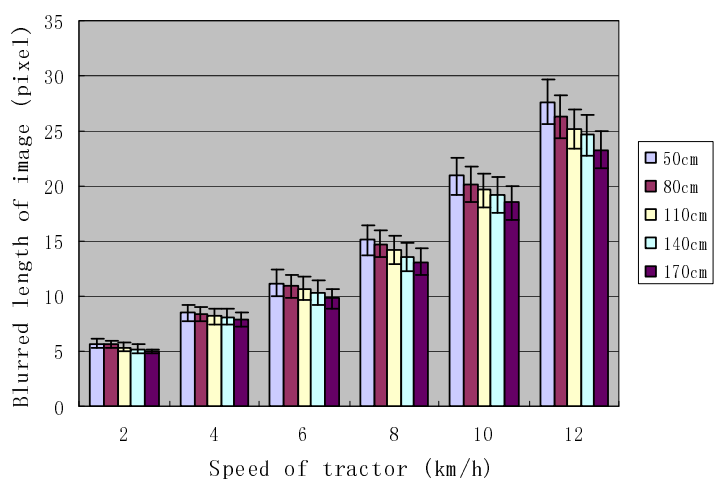

Fig. 3. The relationship of between tractor speed and blur length

Blur angle increased with tractor speed and height of camera increase, the related mathematical model can be expressed as

$$
\theta=a_{0}+a_{1} V+a_{2} H
$$

Where $a 0$ is constant, $a 1, a 2$ is coefficient, $\mathrm{V}$ is tractor speed, $\mathrm{H}$ is height of camera.

Table 1. Parameter value and significance of blur angle

\begin{tabular}{cccc}
\hline & Coefficient & t Stat & P-value \\
\hline$a_{0}$ & -3.52089 & -3.97802 & 0.000469 \\
$a_{1}$ & 1.471714 & 19.61055 & $1.68 \mathrm{E}-17$ \\
$a_{2}$ & 0.021111 & 3.49414 & 0.001658 \\
\hline
\end{tabular}

Table 1 shows that tractor speed and camera angle has an impact on the blur of image with $\mathrm{p}$ value of a1 and a2 less than 0.05 . The value of a1 was more than those of a2, which show that tractor speed has more impact on blur angle than height of camera. The regression equation can be expressed as:

$$
\theta=-3.52089+1.471714 V+0.021111 H
$$

Which correlation coefficient $\mathrm{R} 2=0.94$.

As can be seen from Fig. 3, blur length of image increase with tractor speed increase for the increase of tractor distance in exposure time. Therefore, blur length increased from 5 pixels to 25 pixels while tractor speed changed from 2 to 12 . However, the decrease of shooting range of camera is made when height of camera increased, which cause blur length of image decrease and this trend is evident as tractor speed increase. Therefore, average blur length decrease 0.7 pixels while the height of camera changed from 50 to 170 at tractor speed of 2, but average blur length decreased 4.3 pixels at the speed of 12 . 
Image blur length increase with the increase of tractor speed, but decrease with the increase of camera height, the correlation model can be expressed as:

$$
L=b_{0}+b_{1} V+b_{2} H
$$

Where $b_{0}$ is constant, $b_{1}, b_{2}$ is coefficient, $V$ is tractor speed, and $H$ is height of camera.

Table 2. Parameter value and significance of blur length

\begin{tabular}{cccc}
\hline & Coefficient & t Stat & P-value \\
\hline$b_{0}$ & 1.805778 & 2.049102 & 0.040284 \\
$b_{l}$ & 1.971714 & 26.38732 & $8.18 \mathrm{E}-21$ \\
$b_{2}$ & -0.01556 & -2.58583 & 0.015432 \\
\hline
\end{tabular}

The table 2 shows that tractor speed and camera angle has an impact on the blur length of image with $\mathrm{p}$ value of $b_{1}$ and $b_{2}$ less than 0.05 . The value of $b_{1}$ is more than those of $b_{2}$, which show that tractor speed has more impact on blurring length than height of camera. The regression equation can be expressed as:

$$
L=1.805778+1.971714 \mathrm{~V}-0.01556 \mathrm{H}
$$

Where correlation coefficient $\mathrm{R} 2=0.96$.

In order to obtain maximum reduction of blur image, we must adjust two parameters of tractor speed and camera height. According to the regression equation of blur angle and blur length has obtained, we establish two trend lines. The one trend line is between blurring angle and length with the increase of tractor speed shown in Fig. 4(a), the other line is between blurring angle and length with the increase of camera height shown in Fig. 5(b).

As can be seen from Fig. 4(a), blurring angle and blur length increased while tractor speed became faster, but there is a significant turning point at tractor speed of $8 \mathrm{~km} / \mathrm{h}$. The growth of blur angle and blur length as speed of tractor less than eight is less than growth of those as speed of tractor more than $8 \mathrm{~km} / \mathrm{h}$.

As can be seen from Fig. 4(b), blur angle is increased with the increase of height of camera. On the contrary, blur length is decreased when height of camera increased. There is a point of intersection at $115 \mathrm{~cm}$ heights of camera, which is the lowest point of the sum of blurring angle and blurring length. Therefore, if the height of camera were $115 \mathrm{~cm}$, the image acquisition would keep in lower blur extent.

Based on the above analysis, speed of tractor and height of camera have a significant impact on blur angle and blur length, but speed of tractor has a more significant impact on those than height of camera. In order to obtain maximum reduction of blurred image, tractor speed should not exceed $8 \mathrm{~km} / \mathrm{h}$ and camera height should be around $115 \mathrm{~cm}$.

\subsection{Blurred Image Restoration}

The images captured by the variable rate spraying system based on machine vision are often blurry in some extent. Fig. 10(a) is the blurred image captured by the camera 


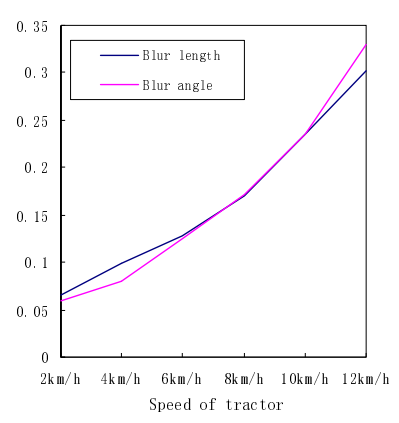

(a)

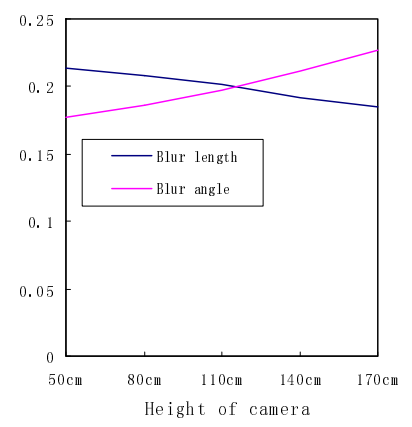

(b)

Fig. 4. The trend of tractor speeds and camera height with blurring length and angle

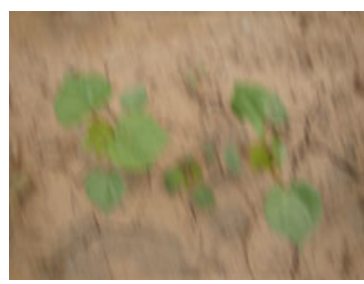

(a)

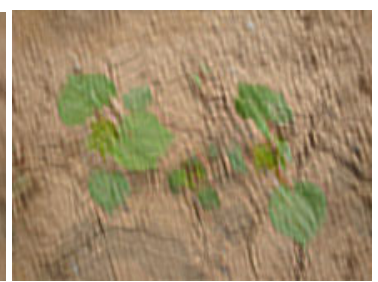

(b)

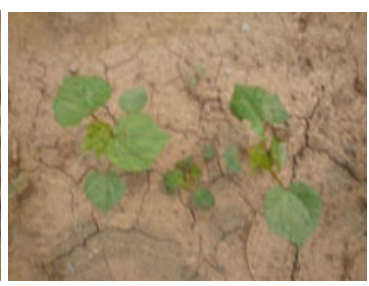

(c)

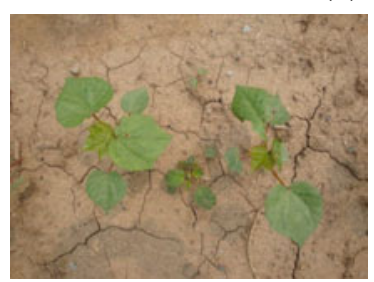

(d)

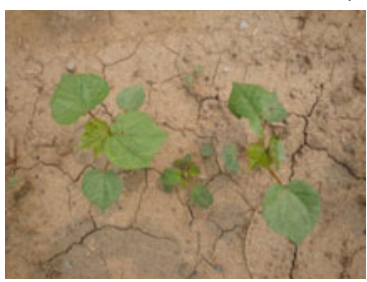

(e)

Fig. 5. Blurred cotton image and recovery image by 4 kinds of algorithms, (a is blurred cotton image, $b$ is image recovered by Blind Algorithm, $c$ is image recovered by L-R algorithm, $d$ is image recovered by Reg algorithm, e is image recovered by Wiener algorithm)

with a velocity of $8 \mathrm{~km} / \mathrm{h}$ and $110 \mathrm{~cm}$ up to the ground, which has 8 degree blur angle and 15 pixel blur length measured by Radon transform. Taking Fig. 10(a) as an object, a comparison is made among the 4 recovery method on the basis of MATLAB7.0, two of them are nonlinear iteration recovery (Blind algorithm and L-R algorithm) and the other two are linear recovery (Reg algorithm and Wiener algorithm). We want to find a proper recovery method to image processing in real-time from recovery effect and calculation time.

Blind Algorithm, L-R algorithm, Reg algorithm and Wiener algorithm four restoration methods Used to recover blurred image Fig. 5(a), among which the iteration frequency of Blind Algorithm and L-R algorithm is 15, the recovery results shown in Fig. 5. The results show that the recovery effect of Reg algorithm and Wiener algorithm 
is better than Blind Algorithm and L-R algorithm, a clear image of cotton fields had been recovered by Reg algorithm and Wiener algorithm. On the contrary, inordinately ripple and circular whorl appeared in the images which were recovered by Blind Algorithm and L-R algorithm for iteration. Therefore, their definition is no better than those of Reg algorithm and Wiener algorithm.

Background segmentation is used to separate the cotton leaf from field soil in the recovery image, which is able to analyze shape and color of cotton leaf after recovery. The image space is transformed from RGB to HSI space, and based on $\mathrm{H}$ variables Otsu threshold algorithm image segmentation is used, which results shown in Fig. 6.

The results of comparison of cotton leaf shape shows that cotton leaves connected, some leaf shape deformed and image distortion is serious in the blurred image. The effect of RL algorithm, Reg algorithm and Wiener algorithm were better than Blind algorithms due to the leaves recovered by which had less linked together and leaf contour clearly. among these three methods, RL algorithm is better than Reg algorithm and Wiener algorithm in noise suppression, so RL algorithm is the best method in recovery of cotton leaf shape.

The results of comparison of cotton leaf color shows that cotton leaf edge section contains a large number of earth colors, which made the error would more if this color were picked up as characteristic variable in the blurred image. After recovery, there were less earth colors on cotton leaf edge in Blind Algorithm and L-R algorithm, while there were no earth colors in Reg algorithm and Wiener algorithm. So Reg algorithm and Wiener algorithm is better than Blind algorithm and L-R algorithm in the recovery effect of cotton leaf color.

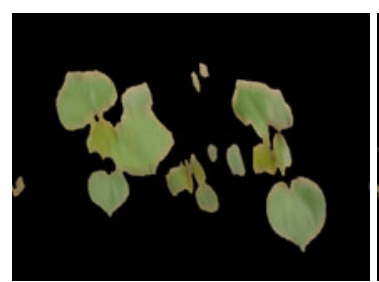

(a)

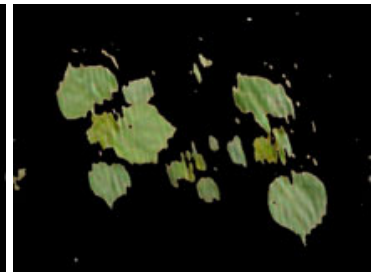

(b)

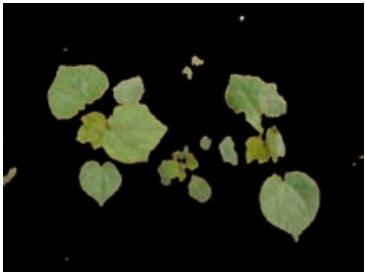

(c)

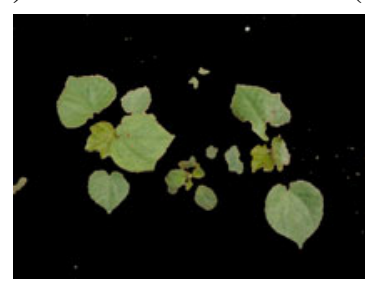

(d)

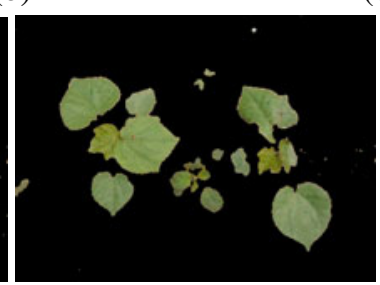

(e)

Fig. 6. Blurred cotton image and 4 kinds of algorithm recovery image after background segmentation

The results of comparison of cotton leaf texture shows that the texture information of blurred images which are made by Prewitt operator on background segment image shown in fig. 7 is lost basically and there is no way to extract its texture information as 
Feature variable. Part of leaf texture is recovered by Blind Algorithm in image, but which is changed and appear some ripple and circular whorl. L-R algorithm is better than Blind algorithm in leaf texture, it recovered the leaf vein normally and there is still some recovery of leaf texture unfinished, while Reg algorithm and Wiener algorithm recovered the texture information completely, leaf vein and rough grain of leaf surface is apparent in image. Reg algorithm and Wiener algorithm is the best in texture.

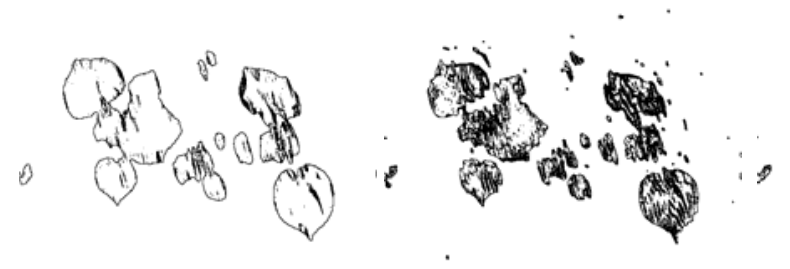

(a) (b)

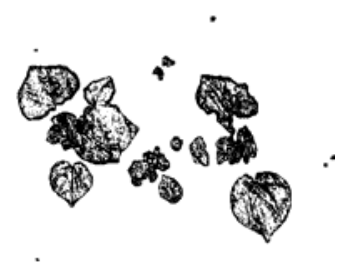

(c)

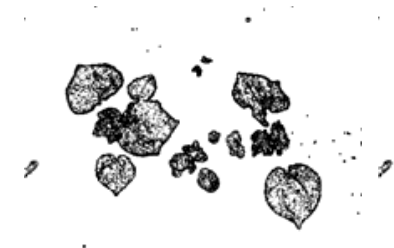

(d)

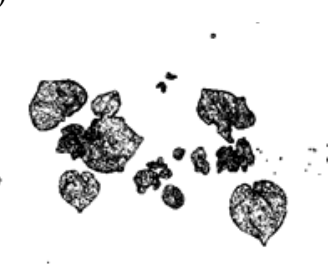

(e)

Fig. 7. Blurred cotton image and 4 kinds of algorithm recovery image after contour extraction

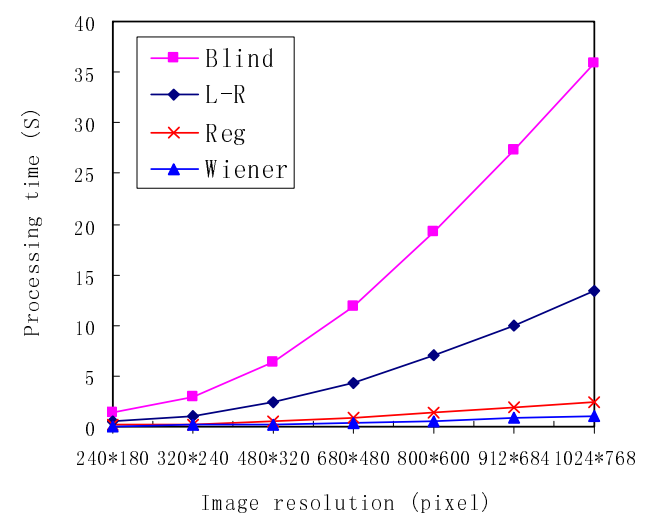

Fig. 8. Computing time of recovery algorithm

The results of computing time on cotton images that computing time of four algorithms increased in some extent with the increase of Image resolution seen from Fig. 8. Computing time of Blind algorithm and L-R algorithm which are belong to non-linear methods increased multiply from $1.3 \mathrm{~s}$ and $0.51 \mathrm{~s}$ to $35.89 \mathrm{~s}$ and $13.35 \mathrm{~s}$ respectively, while computing time of Reg algorithm and Wiener algorithm which are belong to 
linear methods increased slowly from $0.12 \mathrm{~s}$ and $0.05 \mathrm{~s}$ to $2.42 \mathrm{~s}$ and $1.04 \mathrm{~s}$ respectively. In comparison from the same resolution such as $480 * 320$, Wiener algorithm which had a top velocity is $0.19 \mathrm{~s}$, Reg algorithm is 0.50 , while Blind algorithm and L-R algorithm is $6.6 \mathrm{~s}$ and $2.3 \mathrm{~s}$ respectively. Therefore, make a comparison on computing time, Wiener algorithm is at a distinct advantage among the four algorithms.

The test had compared the restoration effect of four recovery methods from color, shape and texture, and compared processing speed from computing time. The results of appraising the four algorithms from the above four aspects with 5 points for each are shown in Table 3. As we can see from the table, the two nonlinear algorithms of Blind algorithm and L-R algorithm got lower points were 7 points and 16 points, while the two linear algorithms of Reg algorithm and Wiener algorithm get higher points were 18 points and 19 points. From the overall evaluation results, Wiener algorithm is the most suitable algorithm for real time image recovery.

Table 3. Appraisal results of image recovery methods

\begin{tabular}{ccccc}
\hline & Blind & L-R & Reg & Wiener \\
\hline Shape & 2 & 5 & 4 & 4 \\
Color & 3 & 4 & 5 & 5 \\
Texture & 1 & 4 & 5 & 5 \\
Time & 1 & 3 & 4 & 5 \\
\hline Total & 7 & 16 & 18 & 19 \\
\hline
\end{tabular}

\section{Conclusion}

According to the practical need of Real-time in the variable rate spraying system, we obtained two groups through this experiment.

The one hand, the speed of Tractor and the height of camera have a important role on the blur extents of real-time image Acquired, and a Regression equations of blur angle and blur length with the speed of tractor and the height of camera is established, which obtained a result that blur degree of real-time image at low levels if the speed of tractor below $8 \mathrm{~km} / \mathrm{h}$ and the height of camera about $115 \mathrm{~cm}$.

The other hand, we compared Wiener algorithm, Reg algorithm, R-L algorithm and Blind algorithm in terms of the shape, color, texture and computing time. The results of comparison show that R-L algorithm got highest scores in the shape for good shape of cotton leaves and less noise. Because of the edge of leaf color no soil color and leaf texture is Recovered Absolutely in image, Reg algorithm and Wiener algorithm is good at the recovering of color and texture. Wiener algorithm on the processing time had obvious advantages, which is much smaller than the other three kinds of image restoration algorithm. Through the above four aspects of evaluation, the result show that Wiener algorithm most suitable for real-time image restoration.

\section{Acknowledgements}

This study has been funded by National 863 Plan sub-project NO: 2008AA100905. 


\section{References}

1. Dobeš, M.: Blurred image restoration: A fast method of finding the motion length and angle. Digital Signal Processing 36, 1-10 (2010)

2. Schuon, S., Diepold, K.: Comparison of motion deblur algorithms and real world deployment. Acta Astronautica 64, 1050-1065 (2009)

3. Ebrahimi, M., Jamzad, M.: Motion blur identification in noisy images using mathematical models and statistical measures. Pattern Recognition 40, 1946-1957 (2007)

4. Lokhande, R.: Identification of parameters and restoration of motion blurred images, pp. 301-305. ACM Press, Dijon (2006)

5. Gonzalez, R.C., Woods, R.E.: Digital Image Processing. 2 version. Restoration new Ruanyu Zhi and translating, pp. 175-176. Electronic Industry Press, Beijing (2003)

6. Li, S.-s.: Real-time motion blur image restoration algorithm. Optics and Precision Engineering 15(5), 767-772 (2007)

7. Gao, Y., et al.: Used as an effective method to restore motion-blurred images. Huaihai Institute of Technology (Natural Science) 18(2), 24-27 (2009)

8. Wang, X., Rongchun: Uniform linear motion blur of the PPS estimates. Computer Applications 21(9), 40-41 (2001)

9. Gui, J., et al.: Based on inverse and singular value decomposition of the exercise of fruit fuzzy image restoration. Biomathematics 21(3), 448-452 (2006)

10. Cai, H., Zhang, Y.-N.: Wang and India and so on. A kind of uniform linear motion blur parameter estimation method. Computer Engineering and Applications 44(19), 175-177 (2008)

11. Chen, X.: The image motion blur restoration technique. Computer and Digital Engineering 36(8), 136-139 (2008)

12. Gao, S., et al.: Motion-blurred image restoration techniques improved algorithm. Communication University of China Natural Science 1(13), 72-76 (2010)

13. Cannon, M.: Blind deconvolution of spatially invariant image blurs with phase. IEEE Trans Acoust Speech Signal Process ASSP-24 (1976)

14. Akira, T., Mitsuji, M., Takao, H.: Median and neural networks hybrid filters. In: IEEE International Conference on Neural Networks, vol. 1, pp. 580-583 (1995)

15. Ben-Ezra, M., Nayar, S.K.: Motion-based motion deblurring. IEEE Transactions on Pattern Analysis and Machine Intelligence 26(6), 689-699 (2004)

16. Gonzalez, R.C., Woods, R.E., Eddins, S.L.: Digital Image Processing Using MATLAB. Pearson Prentice-Hall, Upper Saddle River (2004)

17. Liang, L., Xu, Y.: Adaptive landweber method to deblur images. IEEE Signal Processing Letters 10(5), 129 (2003)

18. Jiang, X., Cheng, D.C., Wachenfeld, S., Rothaus, K.: Motion Deblurring, University of Muenster, Department of Mathematics and Computer Science (2005)

19. Lucy, L.B.: An iterative technique for the rectification of observed distributions. The Astronomical Journal 79(6), 745-754 (1974)

20. Richardson, W.H., et al.: Bayesian-based iterative method of image restoration. Journal of the Optical Society of America 62(1), 55-59 (1972) 